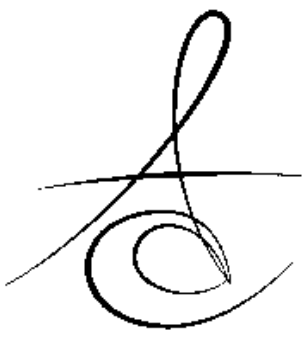

\title{
KADEMELİ ÇOK-TABAKALI/ÇOK RENKLİ ANATOMİK KONTUR ZİRKONYA SERAMİK SİSTEMLERİ İLE SABİT DENTAL PROTEZ UYGULANMASI
}

\section{GRADATIONAL MULTILAYERED/MULTICOLOURED ANATOMIC CONTOUR ZIRCONIA CERAMIC DENTAL PROSTHESES IMPLIFICATION}

\author{
Dr. Öğr. Üyesi Gonca DESTE*
}

Doç. Dr. Rukiye DURKAN*

Makale Kodu/Article code: 2988

Makale Gönderilme tarihi: 09.08.2016

Kabul Tarihi: 02.11.2016

\section{öz}

Kademeli çok-tabakalı (gradational multilayered)/çok renkli (multicoloured) anatomik kontür (anatomic contour) zirkonya seramik sistemleri yeni jenerasyon son teknoloji ürünü ve bilgisayar destekli tasarım/ bilgisayar destekli üretim ile sabit protezlerin yapıldığı materyallerdir. Doğal diş yapısına benzer şekilde servikal, orta üçlü ve insizal bölge renklerinin birlikte bulunduğu mine ve dentin tabakalarından oluşan zirkonya bloklar ile eksternal renklendirme yapılmasına gereklilik olmadan özellikle anterior bölge estetik sabit protezler yapılabilmektedir. Işık geçirgenliğinin yüksek olması, translusent özelliği, yüksek estetik performansı, sertlik, dayanıklılık gibi mekanik özelliklerinin yüksek olması nedeni ile hem anterior hem de posterior bölgede kullanımları bulunmaktadır.

Bu vaka raporunda, maksiler anterior bölgede iki santral dişe yapılan tek kron olarak hazırlanan çok-tabakalı anatomik kontür zirkonya kronlar sunulmaktadır. 1 yıllık kullanım sonunda hastamız, yapılan protezin estetik, renk uyumu, dişeti sağlı̆ı, kolay temizlenebilmesi ve dayanıklığı yönünden bir probleminin olmadığını belirtmiştir.

Anahtar kelimeler: anatomik kontür zirkonya; translusent seramik; çok tabakalı zirkonya

\section{ABSTRACT}

Fixed dental prostheses are produced from gradational multilayered/ multicoloured anatomic contour and zirconia ceramic systems with CAD/CAM. Anterior region aesthetic fixed prostheses can be produced with zirconia blocks which consisting of natural tooth similar cervical, middle triple and incisal region colors. Because of its high mechanical properties such as high light transmittance, translucent property, high aesthetic performance, hardness,durability, it is used in anterior and posterior regions both.

In this case report maxillary anterior anatomical contours prepared as a single multi-layered crowns made of zirconia crowns in two central incisors are offered. At the end of our 1-year patient use, the prosthesis, aesthetic, color harmony, gingival health, easy to clean and in terms of durability stated that there is no problem.

Key words: anatomic countour zirconia, translucent ceramic, multi-layer zirconia

\section{GİRİş}

Monokromatik anatomik kontür zirkonya sabit dental protezler, yüksek estetik, translusensi, hassas tasarım ve üretim teknikleri ile döküm altın restorasyonlara göre düşük maliyet, mekanik özelliklerinin yüksek olması gibi avantajları ile hem estetik olarak anterior hem de posterior bölgede kullanımları giderek yaygınlaşmaktadır. ${ }^{1,2}$

Günümüzde üretilen polikromatik anatomik zirkonya seramik disklerin mekanik özellikleri (eğilme direnci $1100 \mathrm{MPa}$ ) monokromatik anatomik kontür zirkonya ile benzer olmakla birlikte estetik olarak polikromatik zirkonya seramiklerde dört renkli tabaka

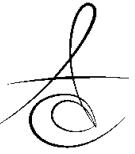


bulunmaktadır (Şekil 1). Sinterizasyon sonrasında eksternal boyamaya intiyaç olmadan politür ve glazür yapıla- bilmektedir. Ayrıca düşük monoklinik faz transfor- masyonu göstermektedir. Tek parça tam kron ve/veya köprü yapılabildiği gibi üzerine düşük ISI veneer seramik uygulaması yapılabilir. ${ }^{3,4}$

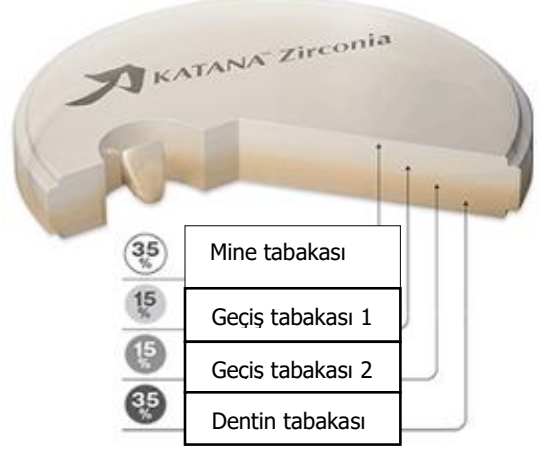

Şekil 1. Çok tabakalı zirkonya disk (KATANA, Noritake Dental Supply Co., Ltd., Miyoshi, Japan)

Zirkonya kron ve köprülerde seramik kalınlığı ve renk tonu materyalden geçen ışık miktarını ve ışık ile polimerize olan rezin simanın polimerizasyon etkinliğini değiştirmektedir. ${ }^{5,6}$ Standart kor (konvansiyonel) zirkonya seramiklerde yaşlanma direncini artırmak için \% 0,25 alümina ilave edilirken yeni jenerasyon anatomik kontür zirkonya seramiklerde alümina oranı $\%$,10,05 düzeyine düşürülerek translusent özellik arttırılmaktadır. Ancak yine de cam seramiklere göre daha az translusensi gösteren semitranslusent bir seramik olduğu rapor edilmektedir. ${ }^{1,2,7}$

Polikristalin zirkonya seramikler standart ve anatomik kontür zirkonya sistemler olarak sınıflandırılmaktadır. Öncelikle zirkonya kor materyali olarak üretilen zirkonya seramikler, metal destekli seramiklerde metal altyapıya bağlı estetik ve biyolojik dezavantajlarından dolayı yüksek oranda tercih edilmişlerdir. ${ }^{8}$ Ancak zirkonya kor materyalin yüksek estetik, biyolojik ve mekanik özelliklerine rağmen üzerinde bulunan seramik üst yapıda oluşan kırılmalar ve ayrılmalar ile birlikte gözlenen zirkonya kor materyali ve veneer porseleni arasındaki termal ekspansiyon farkına bağlı bağlantı problemleri ve opak renkte olmalarından dolayı tek parça halinde restorasyonun yapılabildiği materyal araştırmaları devam etmiştir. ${ }^{8-10}$ $\mathrm{Bu}$ amaç ile üretilen anatomik kontür zirkonya seramik sistemleri ile sabit protetik restorasyonların tek parça halinde yapılması ile klinik başarı ve güvenilirlik sağlanmaktadır. ${ }^{11}$ Ayrıca seramik üst yapı materyali kullanımı gerekmemektedir. ${ }^{2,12}$
Günümüzde zirkonya seramik sistemlerin kullanımlarının artarak devam etmesinde en önemli faktörlerden birisi son teknoloji kullanılarak üretilmiş olmalarıdır. ${ }^{13}$ Bilgisayar destekli tasarım/bilgisayar destekli üretim (CAD/CAM) yöntemleri kullanılarak anatomik kontür zirkonya seramik bloklar ile sabit protetik restorasyonlardan inley, onley, post, lamina, kron, köprü, implant ve abutment restorasyonları yapılmaktadır. ${ }^{14-16}$ Bilgisayar teknolojisinin zirkonya seramiklerin restorasyona dönüştürülmesi işlemlerinde kullanılması ve takiben restorasyonun sinterlenerek üretilmesi ile kullanıma hazır hale gelmektedir.

$\mathrm{Bu}$ vaka raporunun amacl, maksiller santral dişlerdeki estetik olmayan restorasyonların son yıllarda üretilen kademeli çok-tabakalı (gradational multilayered) semisinterize zirkonya seramik kron protezleri ile değiştirilmesi, 1 yıllık kullanım sonunda kronların estetik ve fonksiyonel olarak değerlendirilmesidir.

\section{OLGU SUNUMU}

Afyon Kocatepe Üniversitesi Diş Hekimliği Fakültesi Protetik Diş Tedavisi Anabilim Dalı Klinik-2'ye başvuran 38 yaşındaki kadın hastamızın klinik ve radyolojik muayenesinde maksiller santral dişlerindeki restorasyonların estetik ve fonksiyonel özellik kayıplarının olduğu tespit edilmiştir. Her iki santral dişte bulunan akrilik esaslı tam kronların kırıldığını belirten hastada periodontal bakım sonrasında estetik, fonksiyon, fonetik ve dişeti sağlığı bakımından çok tabakalı anatomik kontür zirkonya seramik sistemi kullanılarak iki adet kron yapılması planlanmıştır (Şekil 2,3).

Hastamıza uygulanılacak tedavi prosedürleri ve seramik materyalin tasarım ve üretimi ile ilgili gerekli bilgiler verildikten sonra bilgilendirilmiş onam formu alınmıştır.

Alınan tanı modellerinde, diş preparasyonlarında yetersiz redüksiyon olduğu için dişlerin labial yüzeylerinde $1 \mathrm{~mm}$, aproksimal yüzeylerde $1 \mathrm{~mm}$, lingual yüzeyde $0,5 \mathrm{~mm}$ ve insizal kenarda $1 \mathrm{~mm}$ redüksiyon olacak şekilde preparasyon düzeltilmiştir. Belirtilen mesafenin mevcut olup olmadığı lateral hareketlerde de kontrol edilmiştir. Preparasyonun servikal sınırları, 1 $\mathrm{mm}$ genişliğinde subgingival oluk (chamfer) basamak şeklinde tasarlanmıştır. Aproksimal duvarlar 5-70 açılandırılarak eğimlendirilmiştir. Servikal marjinlerin, ölçüye net olarak yansıtılması için retraksiyon ipi (Ultrapak, Ultradent, USA) ile gingival retraksiyon yapılarak ölçüsü alınmıştır (Şekil 4). Hastanın diş rengi tespit

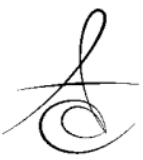


edildikten sonra ölçüler laboratuvara aktarılmışıtır. Hastaya daimi restorasyonları teslim edilinceye kadar fonksiyon, fonasyon ve estetiğin devamını sağlamak amacıyla akrilik materyalden (Protemp Plus, 3M ESPE, $\mathrm{MN})$ geçici restorasyonlar yapılmışır.

Laboratuvar aşamasında, Tip 4 geliştirilmiş sert alçı (Fujirock EP, GC Europa, N.V.) ile güdüklü modeller elde edilmiştir. Sonrasında, alınan kayıtlara göre modeller artikülatöre alınmıştır. Günümüzde kullanımı giderek artan çok tabakalı anatomik kontür zirkonya seramik materyalinden Vita skalası ile uyumlu A1,5-2 renk zirkonya seramik diskten (Katana, Zirconia MultiLayered Disc (ML), Noritake Dental Supply Co., Ltd., Miyoshi, Japonya) CAD/CAM teknolojisi kullanılarak $0.7 \mathrm{~mm}$ kalınlıkta olan ve $\% 20$ oranında büyütülmüş kronlar kazınarak (Novux, NX Mill5), sinterlenmiş (Novux NXS-Plus) 2 üye tam kron elde edilerek glazür işlemi yapılmışııı.

Laboratuvar işlemlerinin tamamlanmasının ardından hasta ağzında lateral ve protrüziv hareketlerde serbestlik sağlanarak, mevcut oklüzyona uygun olduğu anlaşılan kronlar simantasyona hazır hale gelmiştir (Şekil 5,6).

Simantasyon öncesi, siman ve restorasyon ara yüzeyindeki bağlantı kuvvetini arttırmak amacıyla restorasyonun iç yüzeyine $50 \mu \mathrm{m}$ alümina kum içeren cihaz ile 2,8 bar basınç altında $10 \mathrm{~mm}$ uzaklıktan $15 \mathrm{sn}$ süre ile kumlama işlemi (CoJet, 3M ESPE, Seefeld, Almanya) yapılmıştır. Kumlama işleminden sonra restorasyonun iç yüzeyi basınçı su ile 20 sn yıkandıktan sonra 20 sn hava ile kurutulmuştur. Prepare diş yüzeyindeki artıklar temizlenmiştir, bunun haricinde bir işlem uygulanmamıştır. Kronların simantasyonu, ışık ile polimerize olan adeziv rezin siman (NX3, Kerr, ABD) kullanılarak yapılmıştır.

Hastamıza, anatomik kontür zirkonya kronların kullanımı ve hijyeni ile ilgili detaylı bilgi verildikten sonra düzenli kontrollere çağırıımışır.

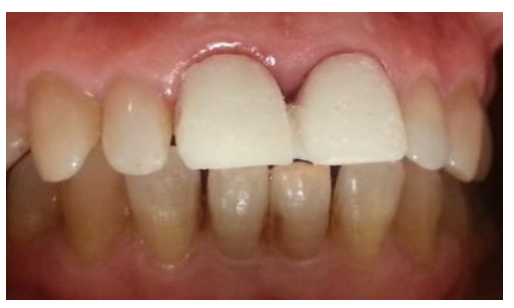

Şekil 2. Hastanın akrilik esaslı tam kronlarının görünümü

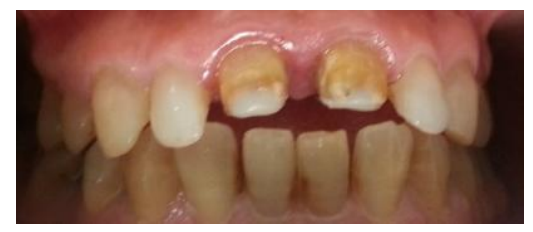

Şekil 3. Akrilik kronlar söküldükten sonraki görünümü

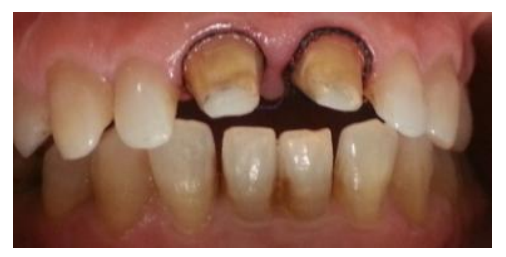

Şekil 4. Preparasyon sonrası gingival retraksiyon

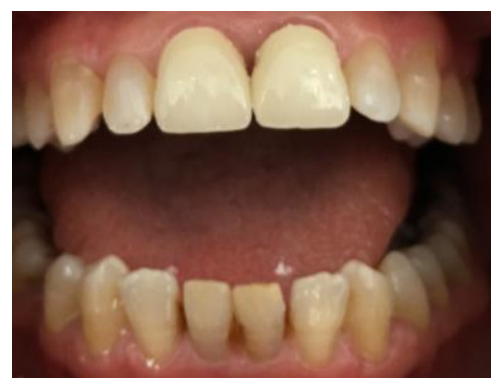

Şekil 5. Çok tabakalı anatomik kontür zirkonya restorasyonlar

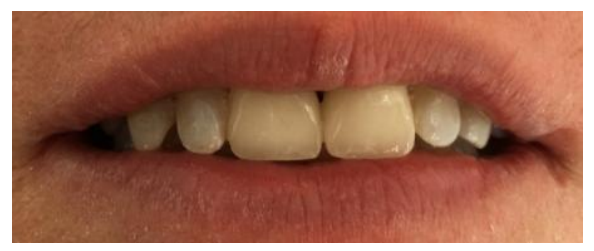

Şekil 6. Restorasyonların bukkal görünümü

\section{TARTIŞMA}

$\mathrm{Bu}$ olgu çalışmasında maksiller santral dişlerin CAD/CAM tekniği ile çok tabakalı anatomik kontür zirkonya sistemi kullanılarak restorasyonu yapılmışır.

Yüksek translusens ve yüksek dayanıklılık en önemli iki parametresini oluşturan anatomik kontür zirkonya seramikler ağız içerisinde bütün sabit restorasyonlarda kullanılabilmektedirler. ${ }^{2,17}$ Anatomik kontür zirkonyanın, geleneksel zirkonyaya göre artmış translusent özelliği sayesinde estetik bölgelerde kullanımı yaygınlaşmışıı. Geleneksel tabakalı restorasyonlarda en sık karşılaşılan komplikasyon olan üst yapı porseleninin alt yapıdan ayrılması ve bunun nedenlerinden biri olan "chipping" problemleri bu

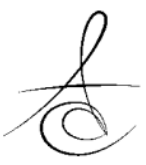




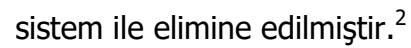

Rinke ve Fischer, anatomik kontür zirkonya sistemlerde translusensinin sınırlı olduğu ve lityum disilikattan daha düşük olduğunu belirtmişlerdir. ${ }^{18}$ Aynı zamanda Beuer ve ark. tarafından yapılan çalışma sonucunda anatomik kontür zirkonyanın veneer seramikzirkonya kor seramik kronlardan daha translusent olduğu belirtilmiştir. ${ }^{19}$

Ueda ve ark. tarafından yapılan bir çalışmada son yıllarda üretilen ve kademeli çok-tabakalı (gradational multilayered) semisinterize zirkonya seramik olarak piyasada bulunan (KATANA, Zirconia MultiLayered Disc (ML), Noritake Dental Supply Co., Ltd., Miyoshi, Japonya) seramik sisteminin konvansiyonel tam kontür zirkonya seramiklerden daha estetik olduğu vurgulanmaktadır. Bu sistemde 3 renk seramik disk üretilmiştir; A-parlak: Vita skalası ile uyumlu, A1,5-2 (corresponds to VITA shade A1.5-2), A-koyu: Vita skalası ile uyumlu, A2,5-3 ve B-parlak: Vita skalası ile uyumlu B1,5-2 şeklinde üretilmiştir. Çok tabakalı zirkonya 4 renk tabakasından oluşmaktadır. Bunlar \% 35 mine, \% 15 ilk kademe tabaka (first gradation layer), \% 15 ikinci kademe tabaka (second gradation layer) ve \% 35 dentin tabakasıdır ${ }^{3}$. Daha sonra sisteme Vita skalasına uygun olarak, C-parlak:C1.5C2), D-parlak (D1.5-D2) ve A-beyaz (0-0.5) ilave edilmiştir. $^{4}$

Bu materyalin ışık geçirgenliğinin değerlendirildiği bir çalışmada $1 \mathrm{~mm}$ kalınlıkta $20 \mathrm{~mm}$ çapında hazırlanan örnekler için mine tabakasının ışık geçirgenliği en yüksek \% 32,8 iken ilk tabaka \% 31,2, ikinci tabaka \% 25,4 ve dentin gövde tabakasının \% 21,7 en düşük olduğu belirlenmiştir. Tabakalarda gözlenen pigmentasyonların ışık geçirgenliğini etkilediği gözlenmiştir. Seramik materyal opak olduğu zaman translusensi parametresi sıfır kabul edilirken, değerin yükselmesi materyalin translusensi özelliğinin arttığını göstermektedir. ${ }^{20}$ Santral dişlerin estetik görünümdeki önemli etkisi bilindiği için çalışmamızda çok tabakalı anatomik kontür zirkonya seramik materyali kullanılmıştır.

Anatomik kontür zirkonya seramiklerde ışık geçirgenliği seramiğin markasına ve seramik kalınlığına göre değişmektedir. Seramik kalınlığı arttıkça ışık geçirgenliği seramik tipine bakılmaksızın azalmaktadır. Ayrıca Katana (Noritake) ve Prettau anterior (Zirkonzahn) seramiklerde ışık geçirgenliği BruxZir seramikten daha yüksek bulunmuştur. ${ }^{1}$
Dört parsiyel stabilize anatomik kontür zirkonya (Prettau, Bruxzir, Zenostar ve Katana) ile bir tam stabilize anatomik kontür zirkonya seramik (Prettau Anterior, Zirkonzahn) ve konvansiyonel zirkonya (ICE Zirkon, Zirkonzahn) 0,5 mm; $1 \mathrm{~mm} ; 1,5 \mathrm{~mm}$ ve $2 \mathrm{~mm}$ olmak üzere farklı kalınlıklarda hazırlanarak spektrofotometre ile ışık geçirgenlikleri karşılaştııılmıştır. Yüzey parlaklıkları polisaj ile artmış ve translusensi değerleri polisaj öncesi 5,65-20,40 arasında iken polisaj sonrasında 5,10-19,95 arasında olmuştur. Translusensi değerleri en düşükten en yüksek değerlere doğru sıralandığı zaman polisaj sonrasında Bruxzir=ICE Zirkon= Prettau <Zenostar $<$ Katana $<$ Prettau Anterior şeklinde bir sıralama elde edilmiştir. $\mathrm{Bu}$ çalışmada Katana zirkonya seramiğin ışık geçirgenliği yüksek bulunmuştur. ${ }^{1}$

Zirkonya sabit protezlerin simantasyonunda yapıştııı simanların tutucululuklarının araştırıldığı in vitro bir çalışmada; rezin siman, rezin modifiye cam iyonomer siman, çinko fosfat siman, polikarboksilat siman, cam iyonomer siman ve geçici siman karşılaştırılmıştır. En iyi tutuculuk değerleri rezin siman ile rezin modifiye cam iyonomer simanda belirlenmiştir. Çinko fosfat siman ile polikarboksilat simanın tutuculuk değerlerinin birbirine eşit ve kabul edilebilir düzeyde olduğu belirtilmiştir. ${ }^{21}$ Cam iyonomer siman ve geçici simanın ise kabul edilemeyecek derecede güçsüz bir bağlantı sağladığı bildirilmiştir., ${ }^{1,22}$ Bizim çalışmamızda da yeterli tutuculuk sağlamak amacıyla estetik kronların simantasyonu ışık ile polimerize olan rezin siman ile yapılmıştır.

Protetik tedavi sonucunda hastamı, kron restorasyonlarının estetik görünümünden memnun kalmıştır. 1 yıllık takip sürecinde estetik ve fonksiyon açısından herhangi bir komplikasyon ile karşılaşılmamıştır. Kontrol seanslarında periodontal dokuların sağlığı iyi ve hastamızın oral hijyeni yeterli görülmüştür

Gonca Deste: ORCID ID: 0000-0002-5481-0063

Rukiye Durkan: ORCID ID: 0000-0002-3237-9997 KAYNAKLAR

1.Sulaiman TA 1, Abdulmajeed AA, Donovan TE, Ritter AV, Vallittu PK, Närhi TO, Lassila LV. Optical properties and light irradiance of monolithic zirconia at variable thicknesses. Dent Mater 2015;31:1180-7.

2.Zhang F, Vanmeensel K, Batuk M, Hadermann J,

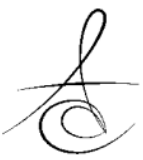


Inokoshi M, Van Meerbeek B, Naert I, Vleugels J. Highly-translucent, strong and aging-resistant 3YTZP ceramics for dental restoration by grain boundary segregation. Acta Biomater 2015; 16: 215-22.

3.Ueda K, Güth JF, Erdelt K, Stimmelmayr M, Kappert $\mathrm{H}$, Beuer F. Light transmittance by a multi-coloured zirconia material. Dent Mater J 2015;34:310-4.

4.KATANA multilayered zirconia now available in new shades [VIDEO] February 06, 2015. www. dentalproductsreport.com. Erişim tarihi: 4.08.2016.

5.Sulaiman TA 2, Abdulmajeed AA, Donovan TE, Ritter AV, Lassila LV, Vallittu PK, Närhi TO. Degree of conversion of dual-polymerizing cements light polymerized through monolithic zirconia of different thicknesses and types. J Prosthet Dent 2015;114:103-8.

6.Rasetto FH, Driscoll CF, Prestipino V, Masri R, von Fraunhofer JA. Light transmission through allceramic dental materials: a pilot study. J Prosthet Dent 2004;91:441-6.

7.Ilie N, Stawarczyk B. Quantification of the amount of blue light passing through monolithic zirconia with respect to thickness and polymerization conditions. J Prosthet Dent 2015;113:114-21.

8.Wang F, Takahashi H, Iwasaki N. Translucency of dental ceramics with different thicknesses. J Prosthet Dent 2013;110:14-20.

9.Fischer J, Stawarzcyk B, Trottmann A, Hammerle $\mathrm{CH}$. Impact of thermal misfit on shear strength of veneering ceramic/ zirconia composites. Dent Mater 2009;25:419-23.

10.Raigrodski AJ, Chiche GJ, Potiket N, Hochstedler JL, Mohamed SE, Billiot S, Mercante DE.The efficacy of posterior three-unit zirconium-oxide-based ceramic fixed partial dental prostheses:a prospective cli- nical pilot study. J Prosthet Dent 2006;96:237-44.

11.Guess PC, Schulthesis S, Bonfante EA, Coelho PG, Ferencz JL, Silva NR. All ceramic systems: laboratory and clinical performance. Dent Clin N Am 2011;55:333-52.

12. Mundhe K, Jain V, Pruthi G, Shah N. Clinical study to evaluate the wear of natural enamel antagonist to zirconia and metal ceramic crowns. J Prosthet Dent 2015; 114:358-63.

13.Batson ER, Cooper LF, Duqum I, Mendonça G. Clinical outcomes of three different crown systems with CAD/CAM technology. J Prosthet Dent 2014; 112: $770-7$.
14.Kohorst $\mathrm{P}$, Junghanns J, Dittmer MP, Borchers L, Stiesch $M$. Different CAD/CAM processing routes for zirconia restorations: influence on fitting accuracy. Clin Oral Investig 2011;15:527-36.

15.Conrad HJ, Seong WJ, Pesun IJ. Current ceramic materials and systems with clinical recommendations: a systematic review. J Prosthet Dent 2007;98:389-404.

16.Larsson C, Vult von Steyern P, Nilner K. A prospective study of implant supported full-arch yttrium-stabilized tetragonal zirconia polycrystal mandibular fixed dental prostheses: three-year results. Int J Prosthodont 2010;23:364-9.

17. Harianawala $\mathrm{HH}$, Kheur MG, Apte SK, Kale BB, Sethi TS, Kheur SM. Comparative analysis of transmittance for different types of commercially available zirconia and lithium disilicate materials. J Adv Prosthodont 2014;6:456-61.

18.Rinke S, Fischer C. Range of indications for translucent zirconia modifications: clinical and technical aspects. Quint Int 2013;44:557-566.

19.Beuer F, Stımmelmayr M, Gueth J F, Edelhoff D, Naumann $M$. In vitro performance of full-contour zirconia single crowns. Dent Mater 2012;28:44956.

20.Villarroel M, Fahl N, Sousa A M, Olıveıra O B. Direct esthetic restorations based on translucency and opacity of composite resins. J Esthet Restor Dent 2011;23: 73-88.

21.Koçak A, Türker Ş B. Diş hekimliğinde zirkonyum. Atatürk Üniv Diş Hek Fak Derg 2006;16:41-45.

22.Zhang Y, Mai Z, Barani A, Bush M, Lawn B. Fracture-resistant monolithic dental crowns. Dent Mater 2016;32:442-9.

\section{Yazışma Adresi}

Doç. Dr. Rukiye Durkan, Afyon Kocatepe Üniveritesi Diş Hekimliği Fakültesi, Protetik Diş Tedavisi Anabilim Dalı, Afyon e-mail:dr.durkan@hotmail.com 